\title{
Hybrid Approach of Relation Network and Localized Graph Convolutional Filtering for Breast Cancer Subtype Classification
}

\author{
Sungmin Rhee ${ }^{1}$, Seokjun Seo ${ }^{1}$, Sun Kim ${ }^{1,2,3}$ \\ ${ }^{1}$ Department of Computer Science and Engineering, Seoul National University \\ ${ }^{2}$ Bioinformatics Institute, Seoul National University, Seoul, Republic of Korea \\ ${ }^{3}$ Interdisciplinary Program in Bioinformatics, Seoul National University \\ lars@snu.ac.kr, dane2522@snu.ac.kr, sunkim.bioinfo@snu.ac.kr
}

\begin{abstract}
Network biology has been successfully used to help reveal complex mechanisms of disease, especially cancer. On the other hand, network biology requires in-depth knowledge to construct diseasespecific networks, but our current knowledge is very limited even with the recent advances in human cancer biology. Deep learning has shown an ability to address the problem like this. However, it conventionally used grid-like structured data, thus application of deep learning technologies to the human disease subtypes is yet to be explored. To overcome the issue, we propose a hybrid model, which integrates two key components 1) graph convolution neural network (graph $\mathrm{CNN}$ ) and 2) relation network (RN). Experimental results on synthetic data and breast cancer data demonstrate that our proposed method shows better performances than existing methods.
\end{abstract}

\section{Introduction}

Breast cancer is one of the most common cancers, especially leading type of cancer in women [Network and others, 2012]. Breast cancer has multiple risk factors for development and proliferation including genetic change, epigenetic change, and environmental factors. Also, breast cancer is a complex, multifactorial disease where interplays between these risk factors decide the phenotype of cancer such as progression, development, or metastasis. Thus, it is a challenging problem to determine how a cancer cell is developed and progressed.

Characterizing mechanisms of a complex disease as a whole is not possible. An effective approach is to define subtypes by dividing cancer into several categories according to various criteria such as phenotype, molecular portraits, and histopathology. Among the several molecular properties based breast cancer subtypes, PAM50 [Parker et al., 2009] has become a standardized model with the clinical utility to make diagnosis decisions in practice or building a treatment plan for a patient. Also, St. Gallen international expert consensus panel introduced a system for recommending adjuvant systemic therapy based on breast cancer subtype since 2011 [Goldhirsch et al., 2013]. However, despite the practical utility of breast cancer subtypes, they are still remained to be suboptimal since the complex mechanism underlying breast cancer cell is not fully investigated.

The main technical issue in elucidating biological mechanisms of breast cancer is that innate relational and cooperative characteristic of genes should be considered. In any specific biological context, multiple dysregulated genes derive phenotypic differences by mechanisms such as forming complexes, regulating each other, or affecting signal transduction. To address the technical difficulties related to complex associations, studies that utilize biological network are needed. Early biological network studies have tried to discover distinct patterns based on edge information [Barabasi and Oltvai, 2004; Barabási et al., 2011], and recent approaches rely on the common paradigm called network propagation, which assumes that the information is propagated to nearby nodes through the edges [Cowen et al., 2017]. However, network biology requires in-depth knowledge to construct disease-specific networks, but our current knowledge is very limited even with the recent advances in human cancer biology.

Deep learning has shown an ability to address the difficult situation like this. However, application of deep learning technologies to the classification of human disease subtypes is not straightforward since deep learning technologies conventionally use grid-like structured data and they are not mainly designed to handle graph data. Recently, graph based deep learning techniques have emerged, which becomes an opportunity to leverage analyses in network biology.

In this paper, we try to advance network bioinformatics by incorporating a novel hybrid method of relation network (RN) and graph convolution neural network (graph CNN). Given the prior knowledge of putative associating genes represented in a graph structure, our proposed method captures localized patterns of associating genes with graph $\mathrm{CNN}$, and then learn the relation between these patterns by RN.

The main contributions of this work are as follows:

- We propose a novel hybrid approach composed of graph CNN and RN. Our method is motivated by the fact that relations between entities are traditionally modelled with a graph structure. To the best of our knowledge, 
this work is the first of its kind.

- We propose a model for biological networks. Since the dimension of the conventional biological network is large, we applied fast graph convolution filtering method that can scale up to the very large dimension. In addition, we modified the relation network to fit in the task.

- We demonstrate the effectiveness of our approach by experiments using synthetic dataset and breast cancer subtype classification data. Our model is able to achieve good performance in terms of both classification evaluation and capturing biological characteristics such as survival hazard and subtype prognosis.

The article is organized as follows. In the next section, we review previous studies related to our work. Then, our model is described in Section 3. In Section 4, we demonstrate the experimental result with synthetic and real dataset.

\section{Related Work}

\subsection{Deep Learnings on Graphs}

Recent survey papers [Niepert et al., 2016; Bronstein et al., 2017] present comprehensive surveys on graph deep learnings that recently emerge. In this section, we review a representative selection of the previous studies related to this work.

In the case of recurrent neural networks (RNN), there has been an attempt [Scarselli et al., 2009] to combine the graph structure with the neural network earlier than CNN. Graph neural network (GNN) is one of such study, which is an extension of recursive neural network and random walk. The representation of each node propagates through edges until it reaches a stable equilibrium. Then it is used as the features in classification and regression problems. This approach is further extended to a method named gated graph sequence neural network (GGS-NN), introducing gated recurrent unit and modifying to output sequence [Li et al., 2015]. Recently, Johnson have proposed a method built upon GGS-NN by allowing graph-structured intermediate representations, as well as graph-structured outputs [Johnson, 2016].

CNN has been successful on domains with underlying grid-like structured data such as computer vision, natural language processing, audio analysis, and DNA sequences. Recently, several works extended CNN to more general topologies like manifolds or graphs [Bruna et al., 2013; Henaff et al., 2015; Niepert et al., 2016; Defferrard et al., 2016]. Bruna et al. have introduced a spectral formulation of graph $\mathrm{CNN}$ (SCNN), which draws on the properties of convolutions in the Fourier domain [Bruna et al., 2013]. They extend the ideas to large-scale classification problems [Henaff et al., 2015]. Nevertheless, the method still does not scale up well due to the computational cost of $\Theta\left(n^{2}\right)$ for matrix multiplication.

The method proposed by Defferrard et al. leverage on the spectral graph CNN (SCNN), which is a key component of our approach. Computational efficiency is improved by using Chebyshev approximation technique. As a result, their method outperforms the existing SCNNs in terms of accuracy in their experiments.

Recently, Graph Attention Network (GAT) is proposed [Veličković et al., 2018], which utilizes masked self-attention mechanism without convolution or RNN to deal with graph structured data. In the method, attention value is evaluated for each of neighboring nodes to produce feature vectors for graph nodes. GAT model has achieved the state-of-art results across four different experiments established on graph structured datasets.

\subsection{Relation Reasoning}

As Google DeepMind's team mentioned in their recent study [Santoro et al., 2017], deductive reasoning methods innately reason about relations of entities from training data, which is also represented as relations [Quinlan, 1990]. However, these approaches lack the ability to deal with fuzzy and variational input data robustly [Harnad, 1990]. Meanwhile in the statistical learning domain, DeepMind recently have proposed a method named relational network as a general solution to relational reasoning in neural networks [Santoro et al., 2017]. The method focuses on relational reasoning with an easy-tounderstand and generalizable network structure, which makes it easy to be modified or combined with other methods. In addition, despite its simplicity in structure, it has demonstrated super-human performance in visual question answering problem. In this paper, we modify the relation network and combine it with graph $\mathrm{CNN}$ for the first time. It shows that the relational reasoning helps to improve the performance of the proposed breast cancer subtype classification task.

\section{Methods}

In this section, we describe the proposed method. Figure 1 illustrates the overall workflow of the proposed method. The first step (Figure $1 \mathrm{~A}$ ) of the method is the graph convolution step to represent and capture localized patterns of the graph nodes (genes). The second step (Figure $1 \mathrm{~B}$ ) is the relational reasoning step. In this step, the model learn the complex association between graph node groups (gene sets) from the learned localized patterns of graph nodes (genes) in the previous step. The next step is to merge the representation of graph convolution layer and relation reasoning layer.

In this paper, we will denote data elements of each sample $p$ as $x_{p} \in R^{n}$, and weighted graph topology as $G=$ $(V, E, A)$, where $V$ and $E$ represent the sets of vertices and edges, respectively. Also, we will use $A$ to denote the weighted adjacency matrix and $N$ to denote the number of vertices, i.e. $|V|$.

\subsection{Localized Pattern Representation by Graph Convolution Neural Network}

For capturing localized patterns of data (gene expression profile), we first mapped input data in the graph structure and used graph CNN technique to find localized patterns of the graph signal. Let $x_{p} \in R^{n}$ be the graph signal (or gene expression) in the sample $p$. Then graph Laplacian matrix $L$ is used to find spectral localized patterns of $x_{p}$ under the graph structure $G$. Laplacian matrix $L$ of graph $G$ is defined as $L=D-A$ where $D$ is a weighted degree matrix, and $A$ is a weighted adjacency matrix of graph $G$.

Then the graph convolution of signal $x$ is defined with graph Laplacian matrix. Let's assume that $L=U \Lambda U^{T}$ is an 


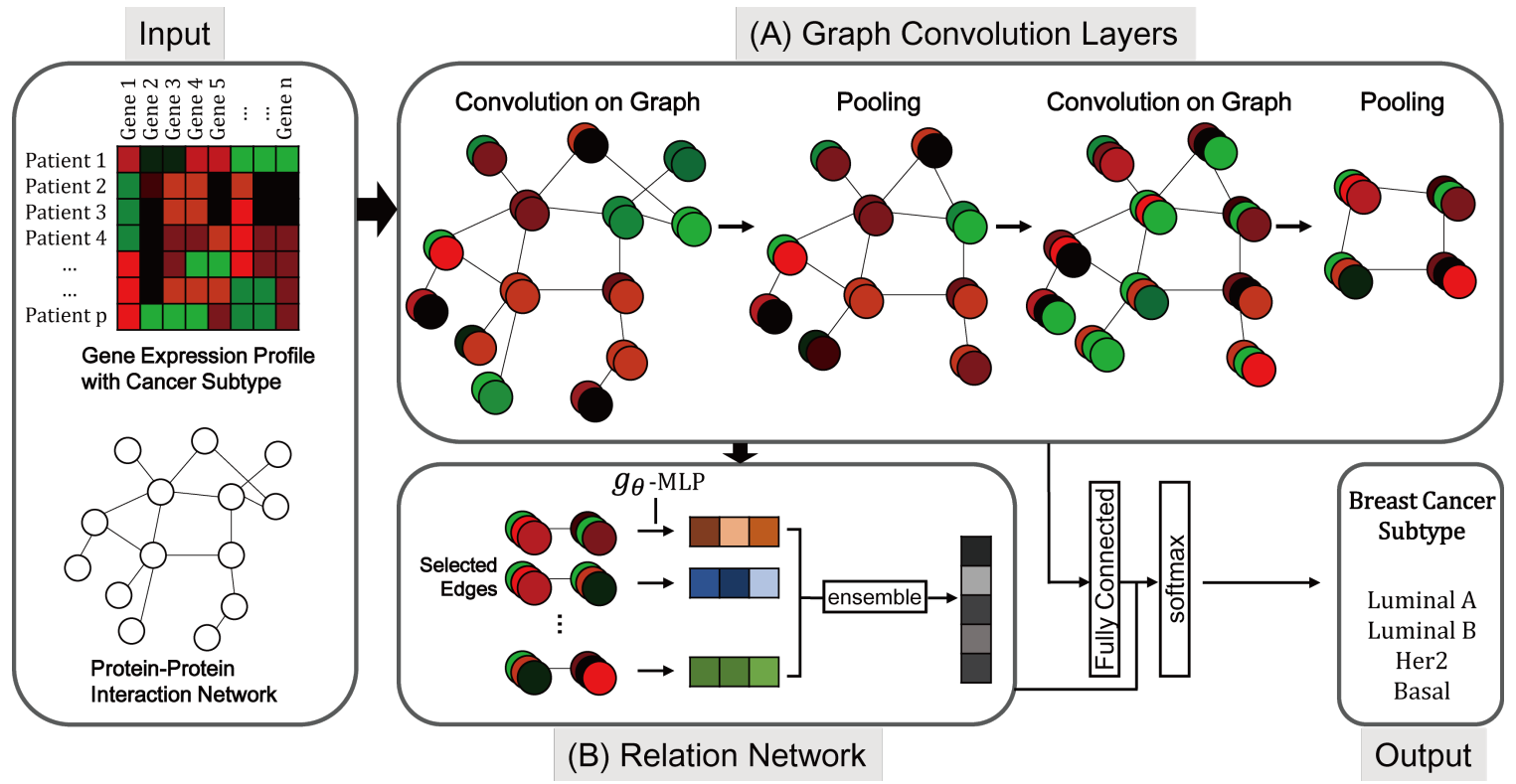

Figure 1: Overview of the proposed method

eigenvalue decomposition of graph Laplacian $L$, where $U=$ $\left[u_{1}, \ldots, u_{n}\right]$ is a matrix composed of eigenvectors $\left\{u_{l}\right\}_{l=1}^{n}$ and $\Lambda$ is a diagonal matrix $\operatorname{diag}\left(\left[\lambda_{1}, \ldots, \lambda_{n}\right]\right)$ composed of eigenvalues $\left\{\lambda_{l}\right\}_{l=1}^{n}$. We can say that $\left\{u_{l}\right\}_{l=1}^{n}$ is a complete set of orthonormal eigenvectors as $L$ is a symmetric positive semidefinite matrix [Defferrard et al., 2016]. Then the graph Fourier transform is defined as $\hat{x}=U^{T} x$ and inverse graph Fourier transform is defined as $x=U \hat{x}$ [Shuman et al., 2013].

Unlike in the classical signal processing domain, it is not straightforward to define the convolution of two signals in spectral graph domain. Thus, convolution theorem in equation 1 is borrowed from classical signal processing domain to define graph convolution as follows,

$$
(x * y)(t)=F^{-1}(F(f) F(g))
$$

where $F$ and $F^{-1}$ denotes Fourier and inverse Fourier transform for each, and $x, y$ denotes two input signals. From the definition of Fourier transform in graph spectral domain, we can induce graph convolution by combining convolution theorem and graph Fourier transform as following equation

$$
\begin{aligned}
x *_{G} y & =U\left(\left(U^{T} x\right) \odot\left(U^{T} y\right)\right) \\
& =U\left(\left(U^{T} y\right) \odot\left(U^{T} x\right)\right) \\
& =U y(\Lambda) U^{T} x
\end{aligned}
$$

where $\odot$ is the element-wise Hadamard product and $y(\Lambda) \in$ $R^{n}$ is a diagonal matrix $\operatorname{diag}\left(\left[\hat{y}\left(\lambda_{1}\right), \ldots, \hat{y}\left(\lambda_{n}\right)\right]\right)$. Since the matrix $U$ is determined by the topology of input graph and invariant, only the matrix $y(\Lambda)$ determines various forms of convolution filters, i.e, elements in $y(\Lambda)$ matrix only are learnable parameters in graph convolution. Among several possible graph convolution filters, our method used polynomial parametrized filter $y_{\theta}(\Lambda)=\sum_{k=0}^{K-1} \theta_{k} \Lambda^{k}$ that can ex- press localized signal patterns in $K$-hop neighboring nodes. However, evaluating polynomial parametrized filter requires very expensive computational complexity $O\left(n^{2}\right)$. To deal with this circumstance, previous study [Hammond et al., 2011] proposed an approximated polynomial named Chebyshev expansion. The Chebyshev polynomial $T_{k}(x)$ of order $k$ is recursively defined as $T_{k}(x)=2 x T_{k-1}(x)-T_{k-2}(x)$ with $T_{0}=1$ and $T_{1}=x$. Then the filter can be approximated as $y_{\theta^{\prime}}(\Lambda)=\sum_{k=0}^{K-1} \theta_{k}^{\prime} T_{k}(\tilde{\Lambda})$ with $\tilde{\Lambda}=2 \Lambda / \lambda_{\max }-I_{n}$.

Going back to the graph convolution in equation 2 , we can now define the final graph convolution filter as

$$
x *_{G} y_{\theta^{\prime}}=\sum_{k=0}^{K-1} \theta_{k}^{\prime} T_{k}(\tilde{L}) x
$$

where $\tilde{L}=2 L / \lambda_{\max }-I_{n}$ is a rescaled graph Laplacian. The equation 3 can be easily induced from the observation $\left(U \Lambda U^{T}\right)^{k}=U \Lambda^{k} U^{T}$. Note that the entire filtering operation only requires $O\left(K|E| F_{\text {in }} F_{\text {out }} P+K|V|\right)$ and is fully differentiable, where $F_{\text {in }}$ and $F_{\text {out }}$ are the number of input and output convolution filters. Thus, entire filtering operation can be learned by backpropagation algorithm [Defferrard et al., 2016].

Convolutioned graph signal is further pooled with neighboring nodes identified by Graclus algorithm [Dhillon et al., 2007] as proposed in the previous study [Defferrard et al., 2016]. There are several pooling strategies in neural network such as max pooling and average pooling. Empirically, the average pooling performed best in our experiments. Therefore, we used average pooling in the proposed method.

\subsection{Learning Relation Between Graph Entities Using Relation Network}

To reason about association between graph nodes, we use relation network $(\mathrm{RN})$, originally defined as 


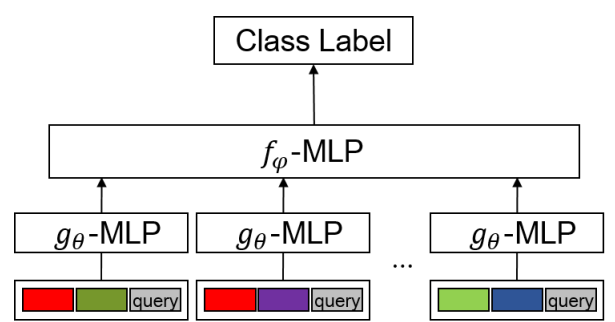

(a) Original RN

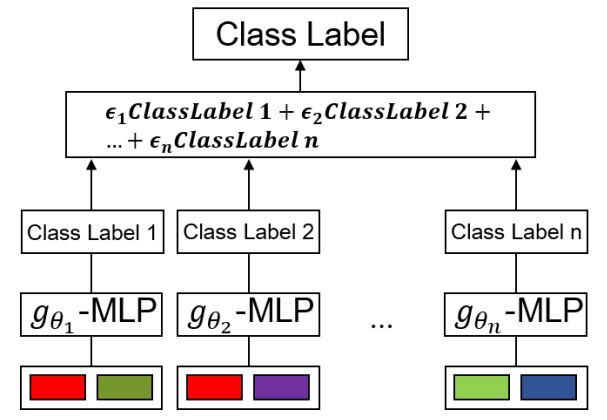

(b) $\mathrm{RN}$ in the proposed method

Figure 2: Architectures of relation networks

$$
R N(O)=f_{\phi}\left(\sum_{i, j} g_{\theta}\left(o_{i}, o_{j}\right)\right)
$$

in the previous study [Santoro et al., 2017] where the input is a set of objects $O=\left\{o_{1}, o_{2}, \ldots, o_{n}\right\}, o_{i} \in R^{m}$. In the original study by Santoro et al., multi-layer perceptron (MLP) is used for function of $f$ and $g$, and the parameters $\theta$ and $\phi$ are synaptic weights of perceptrons. Unlike the task in this paper, there also exists a query for each of the input samples, and each query is embedded to the vector $q$ by an LSTM. Then, Santoro et al. re-define the RN architecture as $R N(O)=f_{\phi}\left(\sum_{i, j} g_{\theta}\left(o_{i}, o_{j}, q\right)\right)$ so that it can process the query in the neural network. This query embedding $q$ can work similarly as an attention mechanism. In other words, query embedding has an ability to select object pairs that are important for the classification task. Also, coordinate values of objects are used to define object pairs in the work of Santoro et al. as it takes an image input, which has innate coordinate information. Therefore, even if all pairs of objects are considered in the original $\mathrm{RN}$, it is able to achieve a good performance.

However, no query information is available in our task and the number of considering objects is larger than the original work. This leads to two technical problems for relation reasoning, 1) object pairs that are not relevant to solve the problem can interfere with learning, and 2) considering all pairs is not feasible as the number of the objects is too large.

To deal with this problem, we have modified the relation network to fit in our task. At first, we sort the edges in the descending order of edge weights. Then top $\kappa$ number of edges are selected as input object pairs to effectively reduce the model size and exclude object pairs that are not highly relevant with the classification problem. Relations in each of

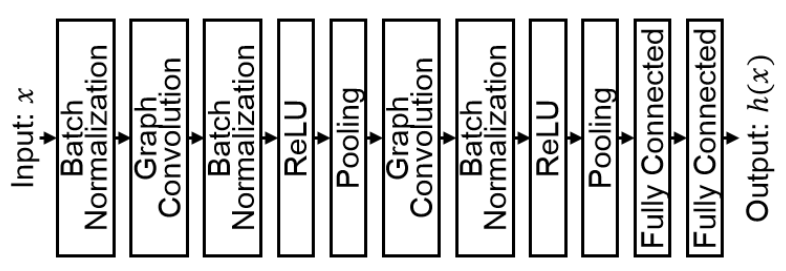

Figure 3: Illustration of $h$ function

the selected pairs are then inferred with $g$ function likewise in original relation network. Unlike the original relation network however, we used different kinds of functions for $g$ and $f$. We use MLPs with separated parameters for each of object pairs other than one MLP with shared parameters as $g$ function. In this way, $g$ functions can learn different relations for each of object pairs. Also, linear ensemble summation is used for $f$ function. The different architectures of original $\mathrm{RN}$ and $\mathrm{RN}$ in the proposed method are illustrated in Figure 2.

The final object relation network is inferred as

$$
R N(O)=\sum_{i, j} \epsilon_{i j} g_{\theta_{i j}}\left(o_{i}, o_{j}\right)
$$

where $\epsilon_{i, j}$ is a learnable parameter that can be interpreted as an attention of each object pairs for the task, $o_{i} \in R^{m}$ is object embedding for each of graph nodes, and $m$ is the number of convolution filter in the last graph convolution layer.

\subsection{Merging Graph Convolution Layer and Relation Network}

The final output of the proposed model architecture is defined as

$$
\hat{y}=\operatorname{softmax}\left(h\left(x_{i}\right)+\sum_{i, j} \epsilon_{i j} g_{\theta_{i j}}\left(o_{i}, o_{j}\right)\right)
$$

to combine outputs from graph convolution layer and relation network, where $h\left(x_{i}\right)$ is defined as a composition of functions; graph convolution, pooling, and fully connected layer.

To be more specific about $h$, we summarize the procedure of $h$ as follows. First, the input signal $x_{i}$ is normalized by a batch normalization method [Ioffe and Szegedy, 2015] to make learning process stable since our dataset has large absolute value and variance. Then, the normalized input signal is filtered by a graph convolution layer as defined in equation 3. Next, the convoluted signal is normalized through a batch normalization method so that the learning process can be accelerated and have regularization effect. Then, ReLU activation function and average pooling are applied. We named the procedure from graph convolution to average pooling as graph convolution layer. After two graph convolution layer, a final feature map is used as an input of fully connected layer. Function $h$ is illustrated in Figure 3.

Output at the last graph convolution layer is also used as a input of the relation network. Then cross-entropy between $\hat{y}$ in equation 6 and classification label is minimized by Adam [Kingma and $\mathrm{Ba}, 2014$ ] algorithm.

Hyperparameters for learning procedure is determined as follows. Two graph convolution layer were used. Each layer 
has 32 convolution filters. $K$ of the first layer is 10 and second layer is 2 . Pooling size is 2 for both of the layers. Two fully connected layers are used with 1024, 512 hidden nodes for each. In the relation network, top 200 and 1000 edges were selected for each of synthetic and real dataset experiment. MLPs for $g$ function have one layer with 128 hidden nodes in synthetic dataset, and 2 layers with 128 hidden nodes for real dataset.

\section{Results and Discussion}

For comparison of performance, we used several methods in experiments of synthetic and real dataset. GCNN denotes graph convolution neural network that has identical hyperparameters with the proposed method. GCNN+RN denotes simple integration of GCNN and vanilla relation network. As we mentioned in methods section, we modify the RN to fit in our task, and GCNN+RN uses original relation network to confirm the effectiveness of RN modification. GAT denotes graph attention network, which is the state-of-the-art neural network that deals with graph structured data. We also compared the method with several baseline methods; RF (Random Forest), kNN (k Nearest Neighbor), SVM (Support Vector Machine), MNB (Multinomial Naive Bayesian), and GNB (Gaussian Naive Bayesian). GNB is used for synthetic dataset since the data is sampled using Multinomial Gaussian Distribution, and MNB is used for real dataset since gene expression data is known to follow multinomial distribution. In real dataset experiment, we also used recent breast cancer classification method based on ensemble SVM [Huang et al., 2017]. The classification performances were measured by a monte-carlo cross validation experiment. We repeatedly sampled $10 \%$ of samples as a validation set and used remaining $90 \%$ of samples as a training set. For each of data splits, the proposed model was fit to training set and accuracy was assessed using validation set. The accuracies were averaged over data splits.

\subsection{Synthetic Experiment}

The first experiment use randomly generated data with different underlying graph structure across classes. The experiment aims to test the ability of model when there are dependencies among attributes (graph vertices) and overlaps among different classes. We used MND (Multivariate Normal Distribution) to generate two class dataset, and each of classes has different covariance matrix to represent different underlying graph structure. We first generated template covariance matrix $\Sigma . \Sigma$ is sampled to have average degree 10 , and each element in $\Sigma$ is sampled with normal distribution, i.e., $\sigma_{i j} \sim \mathcal{N}(0.1,0.1)$ where $\sigma_{i j}$ is an element in $i$ 'th row and $j$ 'th column of $\Sigma$. Then the covariance matrices of two classes, $\Sigma^{1}$ and $\Sigma^{2}$, are generated by randomly deleting or reversing the sign of elements in template covariance matrix. In other words, $\sigma_{i j}^{k}=\mathcal{U}\{-1,1\} \cdot \sigma_{i j}, k \in\{1,2\}$, where $\mathcal{U}$ is the discrete uniform distribution and $k$ is an index for two classes. For $\mu$ in MND of each classes, we both test identical ones and two $\mu^{1}, \mu^{2}$ with small euclidean distances.

Figure 4 shows that our method performs best in all of the experiments except one case with the largest centroid

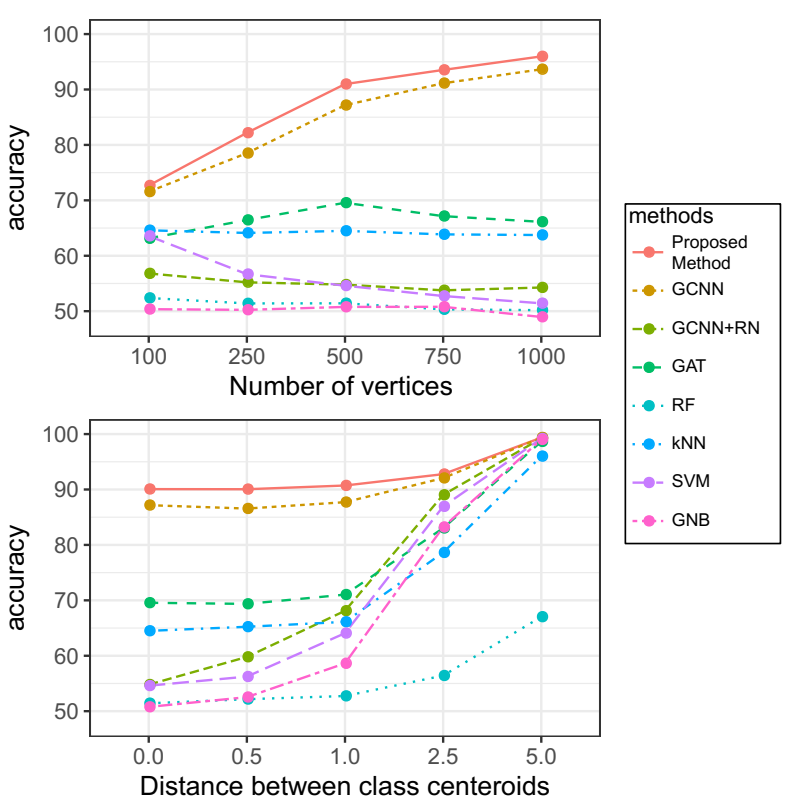

Figure 4: Synthetic data performance comparison. Top plot shows performances across the number of vertices, and bottom plot shows performance across distance between class centroids.

distance. Even in the experiment with the largest centroid distance, performance difference is marginal $(99.35 \%$ by the proposed method, $99.36 \%$ by GCNN). Note that the synthesized dataset is difficult to be classified by approaches like finding hyperplane, since there are large overlap among data points in two classes. Thus, inferring relation between attributes is essential to classify the designed synthetic data. The experiment shows the effectiveness of our method, when dataset that have dependencies among attributes is need to be analysed.

\subsection{Real Dataset}

We applied the proposed method on dataset of human breast cancer patient samples. RNA-seq based expression profiles of genes are extracted from TCGA breast cancer level 3 data [Prat Aparicio, 2012]. There are 57,292 genes in the original expression profile, and we excluded genes that were not expressed and further selected 4,303 genes in the cancer hallmark gene sets [Liberzon et al., 2015] to utilize only genes that are relevant with tumor.

For the classification label of the patient, PAM50 molecular subtype is used. PAM50 is the most commonly used breast cancer subtype scheme. The subtype includes Luminal A, Luminal B, Basal-like, and HER2. Luminal subtype cancer cells are mostly grown from inner (luminal) cells of mammary ducts and known to have better prognoses than other subtypes. Compared to Luminal A however, Luminal B subtype tumors tend to have poorer prognosis factors like higher tumor grade, larger tumor size, and lymph node involvement. Basal-like cancer cells are mostly grown from outer (basal) cells of mammary ducts and known to have worst prognoses and survival rates. HER2 subtype had its name since most HER2 subtype tumors are HER2-positive. HER2 subtype 
Proceedings of the Twenty-Seventh International Joint Conference on Artificial Intelligence (IJCAI-18)

\begin{tabular}{|c|c|c|c|c|}
\hline Methods & $\begin{array}{c}\text { Peak } \\
\text { accuracy }\end{array}$ & $\begin{array}{c}\text { Final } \\
\text { accuracy }\end{array}$ & $\begin{array}{c}\text { F1 } \\
\text { (support) }\end{array}$ & $\begin{array}{c}\text { F1 } \\
\text { (Macro) }\end{array}$ \\
\hline \hline $\begin{array}{c}\text { Proposed } \\
\text { Method }\end{array}$ & $\mathbf{8 6 . 2 9 \%}$ & $\mathbf{8 3 . 1 9 \%}$ & $\mathbf{8 3 . 4 1 \%}$ & $\mathbf{8 2 . 2 6 \%}$ \\
GCNN+RN & $67.65 \%$ & $62.76 \%$ & $62.27 \%$ & $59.88 \%$ \\
GCNN & $85.27 \%$ & $82.39 \%$ & $82.52 \%$ & $81.26 \%$ \\
GAT & $85.64 \%$ & $81.37 \%$ & $81.12 \%$ & $80.15 \%$ \\
SVM & - & $77.53 \%$ & $77.53 \%$ & $76.11 \%$ \\
MNB & - & $75.45 \%$ & $75.87 \%$ & $77.53 \%$ \\
RF & - & $78.54 \%$ & $78.14 \%$ & $75.87 \%$ \\
kNN & - & $62.53 \%$ & $60.22 \%$ & $57.72 \%$ \\
Huang et al. & - & $71.68 \%$ & $70.70 \%$ & $67.93 \%$ \\
\hline
\end{tabular}

Table 1: Performance comparison of the methods on breast cancer subtype classification

tumors tend to have poorer prognoses than luminal subtype tumors. In our study, 338 Luminal A, 265 Luminal B, 149 HER2, and 231 Basal-like patient samples were used for the experiment.

For the topology of the graph, we used STRING protein-protein interaction network [Szklarczyk et al., 2014]. STRING is a curated database of putatively associating genes from multiple pieces of evidence like biological experiments, text-mined literature information, computational prediction, etc.

\subsection{Comparison of Classification Performance}

Table 1 lists accuracies of the proposed model and comparing methods. Peak accuracies during learning processes were listed for top 4 methods in the table, and final accuracies after learning are listed for all of the comparing methods. Also F1 score with two average scheme, weighted by support (the number of true instances for each label) and Macro averaged, are listed. We can see that the proposed method performs best. Also, the simple integration of graph CNN and vanilla $\mathrm{RN}(\mathrm{GCNN}+\mathrm{RN})$ shows the second worst performance. We use identical hyperparameters with the proposed method for GCNN+RN. We believe that GCNN+RN performs poor since, as we described in the method section, the original RN gets query encodings and coordinate values as inputs, that can work as a clue for relevant object selection. However, as there is no coordinate value or query in our task, the changes in our hybrid approach is efficient to make an increase in performance.

\subsection{Consistency of tSNE Visualization and PAM50 Subtype Prognosis}

To qualitatively study whether the learned representation can express the biological characteristic of the patients, tSNE plot [Maaten and Hinton, 2008] of the last convolution feature map is drawn (Right of Figure 5). Only the representation vectors of the objects, which are inputs of relation network, are used to plot. Output of RN is not used since it indicates class labels itself. We can see distinctive patterns between

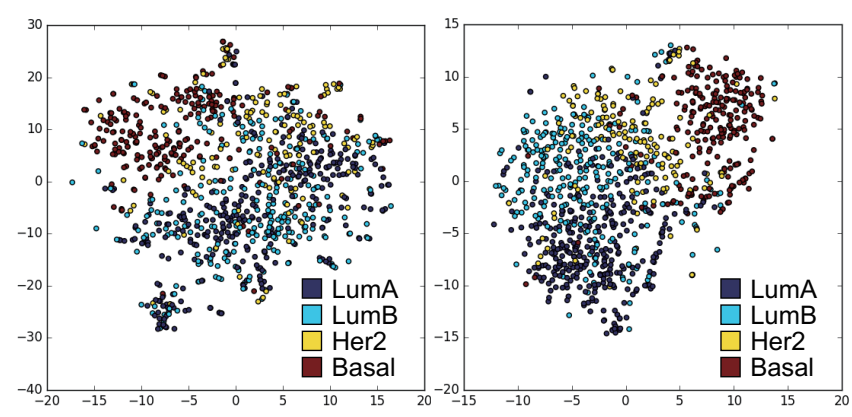

Figure 5: tSNE visualization of graph convolution feature map

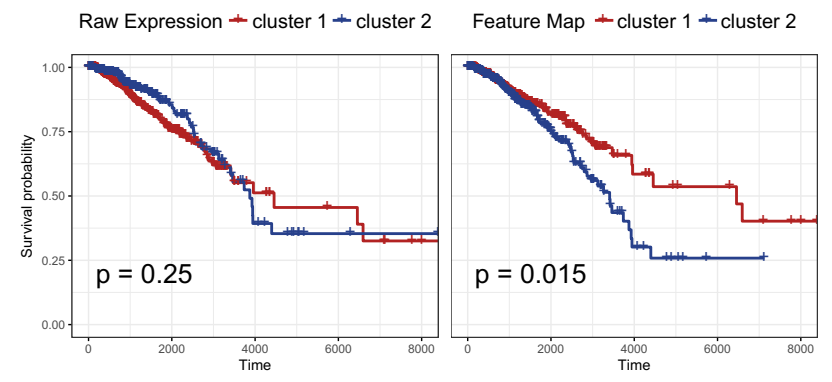

Figure 6: Kaplan meier survival plot of patients

four subtype patients in the plot. However, the distinction between subtypes is not clear than typical examples e.g., tSNE plot of MNIST handwritten digits. We believe that this shows the complexity of the problem we are solving in the task. As we described earlier, the problem has higher input dimension and association between each feature should be considered.

More interestingly, we can see that the order of subtypes in the tSNE plot is identical to the order of prognosis of breast cancer subtypes. It is a well-known fact in the breast cancer clinical domain that Basal-like subtype has the worst prognosis, followed by HER2, Luminal B, and Luminal A. Especially, Basal-like subtype is known to have distinctive molecular characteristics from other subtypes [Bertucci et al., 2012], which is also represented in Figure 5. All of these patterns is not significant in the tSNE plot with raw gene expression (left of Figure 5). Thus, we can say that the proposed method successfully learn the latent molecular properties in the expression profile of the patient samples.

\subsection{Survival Analysis}

To further evaluate the ability of the model to comprehend characteristics of molecular subtypes, we performed survival analysis. We clustered the patients into two groups based on raw gene expression values and feature map data at the last graph convolution layer with dimensions reduced. Agglomerative hierarchical clustering with Ward's criterion [Ward Jr, 1963] is used for clustering and tSNE is used for dimension reduction. Then Kaplan-meier plots (KM plot) [Kaplan and Meier, 1958] drawn for each of two clustering results are seen in Figure 6. KM plot is standard analysis using nonparametric statistics to measure hazard ratios of different patient groups. In medical science, $\mathrm{KM}$ plot is often used to analyze the effectiveness of treatment by comparing KM plot 
of treated and non-treated patient groups.

The plot generated by feature map values (Right of Figure 6) shows that the patient samples are successfully divided into two subgroups that have distinct survival patterns with a p-value smaller than 0.05 , while the plot with raw expression value (Left of Figure 6) failed. This is an interesting result as it shows that the model can simultaneously learn the phenotypic information such as prognosis of the patient while performing the classification task, which is not directly related with the information.

\section{Conclusion}

In this study, we show that hybrid approach of relation network and graph convolution neural network can learn the complex molecular mechanisms underlying breast cancer cells. The proposed method is designed to perceive cooperative patterns of genes and their associations. We observed that the method is successful to capture molecular characteristics of breast cancer in both quantitative and qualitative evaluation. We anticipate that our approach can extend the territory of both network bioinformatics and deep learnings. One important future work of the method is to extend the model to manage multiple heterogeneous data sources like sRNA sequencing, DNA methylation, as well as gene expression data. To do this, we plan to extend the model by incorporating other techniques such as multi-view learning and/or transfer learning.

\section{Acknowledgments}

This research is supported by Next-Generation Information Computing Development Program through the National Research Foundation of Korea(NRF) funded by the Ministry of Science, ICT(No.NRF-2017M3C4A7065887), the Collaborative Genome Program for Fostering New Post-Genome Industry of the National Research Foundation (NRF) funded by the Ministry of Science and ICT (MSIT) (No.NRF2014M3C9A3063541), and a grant of the Korea Health Technology R\&D Project through the Korea Health Industry Development Institute (KHIDI), funded by the Ministry of Health \& Welfare, Republic of Korea (grant number : HI15C3224).

\section{References}

[Barabasi and Oltvai, 2004] Albert-Laszlo Barabasi and Zoltan N Oltvai. Network biology: understanding the cell's functional organization. Nature reviews. Genetics, 5(2):101, 2004.

[Barabási et al., 2011] Albert-László Barabási, Natali Gulbahce, and Joseph Loscalzo. Network medicine: a network-based approach to human disease. Nature reviews. Genetics, 12(1):56, 2011.

[Bertucci et al., 2012] F Bertucci, P Finetti, and D Birnbaum. Basal breast cancer: a complex and deadly molecular subtype. Current molecular medicine, 12(1):96-110, 2012.
[Bronstein et al., 2017] Michael M Bronstein, Joan Bruna, Yann LeCun, Arthur Szlam, and Pierre Vandergheynst. Geometric deep learning: going beyond euclidean data. IEEE Signal Processing Magazine, 34(4):18-42, 2017.

[Bruna et al., 2013] Joan Bruna, Wojciech Zaremba, Arthur Szlam, and Yann LeCun. Spectral networks and locally connected networks on graphs. arXiv preprint arXiv:1312.6203, 2013.

[Cowen et al., 2017] Lenore Cowen, Trey Ideker, Benjamin J Raphael, and Roded Sharan. Network propagation: a universal amplifier of genetic associations. Nature Reviews Genetics, 2017.

[Defferrard et al., 2016] Michaël Defferrard, Xavier Bresson, and Pierre Vandergheynst. Convolutional neural networks on graphs with fast localized spectral filtering. In Advances in Neural Information Processing Systems, pages 3844-3852, 2016.

[Dhillon et al., 2007] Inderjit S Dhillon, Yuqiang Guan, and Brian Kulis. Weighted graph cuts without eigenvectors a multilevel approach. IEEE transactions on pattern analysis and machine intelligence, 29(11), 2007.

[Goldhirsch et al., 2013] Aron Goldhirsch, Eric P Winer, AS Coates, et al. Personalizing the treatment of women with early breast cancer: highlights of the st gallen international expert consensus on the primary therapy of early breast cancer 2013. Annals of oncology, 24(9):2206-2223, 2013.

[Hammond et al., 2011] David K Hammond, Pierre Vandergheynst, and Rémi Gribonval. Wavelets on graphs via spectral graph theory. Applied and Computational Harmonic Analysis, 30(2):129-150, 2011.

[Harnad, 1990] Stevan Harnad. The symbol grounding problem. Physica D: Nonlinear Phenomena, 42(1-3):335-346, 1990.

[Henaff et al., 2015] Mikael Henaff, Joan Bruna, and Yann LeCun. Deep convolutional networks on graph-structured data. arXiv preprint arXiv: 1506.05163, 2015.

[Huang et al., 2017] Min-Wei Huang, Chih-Wen Chen, WeiChao Lin, Shih-Wen Ke, and Chih-Fong Tsai. Svm and svm ensembles in breast cancer prediction. PloS one, 12(1):e0161501, 2017.

[Ioffe and Szegedy, 2015] Sergey Ioffe and Christian Szegedy. Batch normalization: Accelerating deep network training by reducing internal covariate shift. In International Conference on Machine Learning, pages 448-456, 2015.

[Johnson, 2016] Daniel D Johnson. Learning graphical state transitions. 2016.

[Kaplan and Meier, 1958] Edward L Kaplan and Paul Meier. Nonparametric estimation from incomplete observations. Journal of the American statistical association, 53(282):457-481, 1958.

[Kingma and Ba, 2014] Diederik Kingma and Jimmy Ba. Adam: A method for stochastic optimization. arXiv preprint arXiv:1412.6980, 2014. 
[Li et al., 2015] Yujia Li, Daniel Tarlow, Marc Brockschmidt, and Richard Zemel. Gated graph sequence neural networks. arXiv preprint arXiv:1511.05493, 2015.

[Liberzon et al., 2015] Arthur Liberzon, Chet Birger, Helga Thorvaldsdóttir, Mahmoud Ghandi, Jill P Mesirov, and Pablo Tamayo. The molecular signatures database hallmark gene set collection. Cell systems, 1(6):417-425, 2015.

[Maaten and Hinton, 2008] Laurens van der Maaten and Geoffrey Hinton. Visualizing data using t-sne. Journal of Machine Learning Research, 9(Nov):2579-2605, 2008.

[Network and others, 2012] Cancer Genome Atlas Network et al. Comprehensive molecular portraits of human breast tumors. Nature, 490(7418):61, 2012.

[Niepert et al., 2016] Mathias Niepert, Mohamed Ahmed, and Konstantin Kutzkov. Learning convolutional neural networks for graphs. In International Conference on Machine Learning, pages 2014-2023, 2016.

[Parker et al., 2009] Joel S Parker, Michael Mullins, Maggie CU Cheang, Samuel Leung, David Voduc, Tammi Vickery, Sherri Davies, Christiane Fauron, Xiaping He, Zhiyuan $\mathrm{Hu}$, et al. Supervised risk predictor of breast cancer based on intrinsic subtypes. Journal of clinical oncology, 27(8):1160-1167, 2009.

[Prat Aparicio, 2012] Aleix Prat Aparicio. Comprehensive molecular portraits of human breast tumours. Nature, 2012, vol. 490, num. 7418, p. 61-70, 2012.

[Quinlan, 1990] J Ross Quinlan. Learning logical definitions from relations. Machine learning, 5(3):239-266, 1990.

[Santoro et al., 2017] Adam Santoro, David Raposo, David G Barrett, Mateusz Malinowski, Razvan Pascanu, Peter Battaglia, and Tim Lillicrap. A simple neural network module for relational reasoning. In Advances in Neural Information Processing Systems 30, pages 4974-4983. 2017.

[Scarselli et al., 2009] Franco Scarselli, Marco Gori, Ah Chung Tsoi, Markus Hagenbuchner, and Gabriele Monfardini. The graph neural network model. IEEE Transactions on Neural Networks, 20(1):61-80, 2009.

[Shuman et al., 2013] David I Shuman, Sunil K Narang, Pascal Frossard, Antonio Ortega, and Pierre Vandergheynst. The emerging field of signal processing on graphs: Extending high-dimensional data analysis to networks and other irregular domains. IEEE Signal Processing Magazine, 30(3):83-98, 2013.

[Szklarczyk et al., 2014] Damian Szklarczyk, Andrea Franceschini, Stefan Wyder, et al. String v10: proteinprotein interaction networks, integrated over the tree of life. Nucleic acids research, 43(D1):D447-D452, 2014.

[Veličković et al., 2018] Petar Veličković, Guillem Cucurull, Arantxa Casanova, Adriana Romero, Pietro Liò, and Yoshua Bengio. Graph attention networks. In 6th International Conference on Learning Representations, 2018.
[Ward Jr, 1963] Joe H Ward Jr. Hierarchical grouping to optimize an objective function. Journal of the American statistical association, 58(301):236-244, 1963. 